\title{
Malnutrition According to GLIM Criteria Acts as a Risk Factor for Survival of Nasopharyngeal Carcinoma Patients Undergoing Radiotherapy : A Retrospective Study Highlighting the Nutritional Management in Radiotherapy
}

\section{Meng Wan}

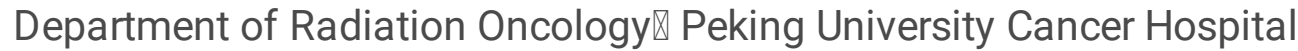

\section{Lichuan Zhang}

School of Nursing, Peking University

\section{Changshun Chen}

Department of Radiation Oncology, First Hospital of Tsinghua University

\section{Dan Zhao}

Department of Radiation Oncology, Peking University Cancer Hospital

\section{Baomin Zheng}

Department of Radiation Oncology, Peking University Cancer Hospital

\section{Shaowen Xiao}

Department of Radiation Oncology, Peking University Cancer Hospital

Weixin Liu

Department of Radiation Oncology, Peking University Cancer Hospital

\section{Xiaolong Xu}

Department of Radiation Oncology, Peking University Cancer Hospital

\section{Yujie Wang}

Peking University School of Nursing

\section{Bing Zhuang}

Peking University School of Nursing

\section{Tong Zhang}

Peking University School of Nursing

Sanli Jin

Peking University School of Nursing

\section{Weihu Wang}

Department of Radiation Oncology, Peking University Cancer Hospital

Qian Lu

Peking University School of Nursing 


\section{Yan Sun ( $\square$ lisa_sun67@126.com )}

Peking University Cancer Hospital and Institute

\section{Research}

Keywords: Nasopharyngeal carcinoma, Radiotherapy, Malnutrition, GLIM, Adverse events, Survival

Posted Date: January 19th, 2021

DOI: https://doi.org/10.21203/rs.3.rs-147693/v1

License: (c) (1) This work is licensed under a Creative Commons Attribution 4.0 International License. Read Full License 


\section{Abstract}

\section{Background}

Nutritional status of nasopharyngeal carcinoma (NPC) patients lying at a high risk of nutrition defect to a great extent determines the post-treatment outcomes, which deserves thorough observation. However, limited ever-conducted studies regarding the NPC patients' malnutrition by effective methods like the Global Leadership Initiative in Malnutrition (GLIM) criteria heavily obstructed this prospect. Our study aimed to investigate the incidence of malnutrition in NPC cancer patients using GLIM criteria and explore the relationships between malnutrition and adverse effects, survival status.

Methods

A retrospective chart review of 113 patients with NPC from December 2016 to October 2019. The nutritional status of NPC undergoing radiotherapy (RT) was determined by GLIM criteria at three-time points: pre-RT, in the middle of RT, and at the end of RT. The adverse effects were graded according to the Radiation Therapy Oncology Group (RTOG) scoring criteria. Kaplan-Meier method was used to compare survival outcomes, and the differences were evaluated using the log-rank test.

Results

Nineteen cases (16.8\%) were malnutrition before radiotherapy; overall stage $(p=0.017)$ and induction chemotherapy $(p=0.002)$ contributed to pre-radiotherapy malnourishment, and the nutritional status worsened with radiotherapy. Of the phenotypic GLIM criteria, low fat free mass index (FFMI) before radiotherapy was associated with mucositis and radiodermatitis $(p<0.05)$. With a median follow-up of 28.3 months, the Kaplan-Meier curve indicated that malnutritional patients had significantly poorer Progress Free survival (PFS); 2-year PFS was $88.9 \%$ in the well-nourishment group vs. $62.1 \%$ in the malnutrition group $(p=0.015)$. In multivariate analysis, nutritional status and gender were independent prognostic factors of poorer PFS (HR 3.387, 95\% Cl 1.299-8.834, $p=0.013$ and HR 5.698, 95\% Cl 1.290$25.168, p=0.022)$.

\section{Conclusion}

Malnutrition is common in NPC patients, and it increases significantly during radiotherapy. Malnutrition, according to GLIM criteria, is correlated with survival. Our study could justify the effectiveness and usability of GLIM for NPC patients undergoing RT. Appropriate nutritional guidance or nutritional support should be taken to improve the NPC patients' nutritional status during radiotherapy.

\section{Background}

Nowadays, nasopharyngeal carcinoma (NPC) prevails, especially in southeastern Asia and the southern region of China, with relatively steady prevalence [1]. Due to unresectable characteristics resulting from less approachable anatomy regions of the nasopharynx and proximity to vital organs, radiotherapy 
predominates among the repertoire of cancer curative strategies with considerably acceptable outcomes emanating longevity survival period and comparatively low mortality [2]. Nevertheless, radiotherapy dichotomizes the corresponding clinical outcomes, bringing about not only the clinically beneficial effects but the condition-worsening pernicious complications as highly-frequent onsets of mucositis, dysphagia, nausea, anorexia, and other adverse events [3-5], among which mucositis features as one of the major radiotherapy-linked adverse effects, inversely impeding the food intake and interrupting the radiotherapy $[6,7]$. Furthermore, it has also been substantialized that declined nutritional status interplays with the higher risk of mortality in head and neck cancer, potentially engendered by related adverse events, which has been proven in some reports [8, 9]. For NPC patients, affected organs at risk by radiotherapy treatment relate to feeding disturbance, accounting for weight loss, and even survival $[6,10]$. Therefore, the NPC patients' malnourishment remains unignorable as the occurrence has been reported to range from $13-43 \%$, showing a threat to NPC patients who receive radiotherapy [11-13].

Regarding malnutrition, more complicated than what it shows on the face value, for a long term, the definition upon it varies in terms of criteria adapted and parameters included, plus, stands devoid of comprehensively unified standardization instead the divided diagnostic guidelines [14-16]. The Global Leadership Initiative in Malnutrition (GLIM) has constantly conducted efforts on clarifying malnutrition, and recent updates had been released [17], which advances further the definition on malnutrition. Unfortunately, the analysis of NPC patients' malnutrition by GLIM criteria remained less reported, and associations underlying the nutrition shortage and post-treatment adverse effects were kept elusive hitherto. Besides, the GLIM criteria require more evidence and studies performed in different cancers to verify its effectiveness and feasibility, which, conclusively, forms the main objective and backbone of our study.

\section{Methods}

\section{Participants}

The study participants were NPC patients who were newly diagnosed and treated at a tertiary cancer hospital in Beijing, with or without induction chemotherapy, from December 2016 to October 2019. All patients underwent complete medical history, physical and neurological examinations, direct fiber-optic nasopharyngoscopy, MRI scan of skull to sternoclavicular joint, chest radiography or $\mathrm{CT}$, abdominal sonography, and whole-body bone scans. PET-CT was performed to evaluate metastasis if clinically indicated. All patients received routine dental evaluations before radiotherapy. According to the 8th edition of the International Union against Cancer/American Joint Committee on Cancer (UICC/AJCC) tumor-node-metastasis system, the overall stage of every patient was re-staged.

Other inclusion criteria for this study included age over 18 years; performance status was stratified according to Eastern Cooperative Oncology Group (ECOG) as 0 (fully activated) or 1 (restricted); adequate hematologic, hepatic, renal, and gastrointestinal functions to receive radiotherapy or chemoradiotherapy; have no cognitive and communication impairments. Exclusion criteria were listed as follows: distant 
metastases; patients with relapse of nasopharyngeal cancer; other secondary tumors; prior chemoradiotherapy treatment within the last six months; restricted mobility or unconsciousness induced pharmacologically leading to be unable to be measured.

\section{Clinical Treatments}

Gross tumor volume (GTV) included the primary nasopharyngeal carcinoma lesion (GTVnx) and regional lymph node metastases (GTVnd). Clinical tumor volume (CTV) was divided into two parts: CTV1 indicated the high-risk region, which covered $0.5 \mathrm{~cm}$ around the GTVnx and included the areas of nasopharyngeal mucosa and $0.5 \mathrm{~cm}$ below the nasopharyngeal mucosa; and CTV2 indicated the low-risk region. The expanding margins of the planning target volume (PTV) were defined as $3 \mathrm{~mm}$. All patients were treated with intensity-modulated radiation therapy (IMRT) in a schedule of 33 fractions, five days per week. The prescribed radiotherapy dose was 70 Gy to 95\% PGTVnx and 95\% PGTVnd, 60-66Gy to 95\% PTV1, and 54-60Gy to $95 \%$ PTV2.

Radiotherapy alone was usually recommended for the patients with stage I, concurrent chemotherapy (CCRT) for stage II, and CCRT with or without induction chemotherapy (IC) for stage III-IVA.

Induction chemotherapy consisted of platinum-based regimens, including taxanes plus platinum with fluorouracil, taxanes with platinum, fluorouracil with platinum, and gemcitabine with platinum every three weeks for 1-3 cycles. CCRT was administered tri-weekly combined with cisplatin or nedaplatin (100 $\mathrm{mg} / \mathrm{m}^{2}$ or $80 \mathrm{mg} / \mathrm{m}^{2}$, according to patients' health condition).

\section{Nutritional assessment}

Nutritional risk was assessed according to the Nutritional Risk Screening 2002 (NRS-2002), which was proposed by Kondrup $\mathrm{J}$ et al. in 2003 [18]. It considers the severity of disease (mild, moderate, or severe) and impaired nutritional status (low, moderate, or severe), with an adjustment for the age of $\geq 70$ years. For the section on "severity of the disease", patients were scored according to the individual medical history. For "impaired nutritional status", patients were scored according to weight, body mass index (BMI), or food intake change. The final scoring of NRS-2002 ranges from 0-7; a score $\geq 3$ denotes nutritional risk [19].

Patients were assessed for malnutrition using the GLIM criteria, which used a two-step-model for risk screening and diagnosis assessment, where the first step is to identify patients who are at nutritional risk with NRS-2002. Secondly, patients who meet at least one of the phenotypic and one of the etiologic criteria of malnutrition are identified [17]. In our study, phenotypic criteria included weight loss, body mass index (BMI), and reduced muscle mass including fat free muscle index (FFMI) and appendicular skeletal muscle mass index (ASMI); etiologic criteria included reduced food intake and disease burden, because in our hospital, detection indicators of inflammation, such as CRP was not measured in all patients, it was not included in this analysis. 
The patient's weight six months before radiotherapy was self-reported or retrieved from patient files when available, whereas health professionals scaled the current weight at admission.

Body composition was measured by body composition analyzer InBody 120 , which relied upon bioelectrical impedance analysis (BIA) technology, with $20 \mathrm{kHz}$ and $100 \mathrm{kHz}$, with eight electrodes distributed in the handle and foot bottom, and data acquired by the procedures of the patient barefoot standing on the electrode, holding the handle with both hands to put on the body side. Eventually, data including weight, body mass, BMI, fat-free mass (FFM), appendicular skeletal muscle mass (ASM), and fat mass (FM) could be generated. The FFMI and ASMI were calculated by dividing a patient's FFM or ASM values by the height squared $\left(\mathrm{m}^{2}\right)$. FFMl $<17 \mathrm{Kg} / \mathrm{m}^{2}$ (male) or $<15 \mathrm{Kg} / \mathrm{m}^{2}$ (female) signified low FFMI, ASMI $<7 \mathrm{Kg} / \mathrm{m}^{2}$ (male) or $<5.7 \mathrm{Kg} / \mathrm{m}^{2}$ (female) was low ASMI [17] .

\section{Nutritional Intervention}

The nutritional intervention plan (except dietary counseling) was not routinely scheduled before treatment for any patients. For patients with slightly poor appetite or mucositis but who had normal swallowing function, we recommended oral supplements or enteral nutrition; however, for patients with severe pain who were able to swallow, we recommended Fentanyl Transdermal Patches (Janssen Pharmaceutica N.V.) to relieve pain. Parental nutrition was delivered for three consecutive days when patients could not intake enough energy by enteral nutrition. No one received a nasogastric tube (NG) or percutaneous gastrostomy (PEG).

\section{Side effects of (chemo)radiotherapy}

The adverse effects during radiotherapy were detected weekly; acute toxicities were graded according to the Radiation Therapy Oncology Group (RTOG) scoring criteria. Radiotherapy-related side effects in the participants were assessed based on these symptoms, including dermatitis, dry mouth, mucositis, loss of taste, loss of appetite, nausea, difficulty swallowing. Chemotherapy-related side effects, including hematologic and non-hematologic toxicity. The worst adverse effects of each patient were selected for subsequent analysis.

\section{Data Collection}

Clinical data were retrospectively collected from their medical records, like age at diagnosis, gender, height, history of tobacco smoking alcohol consumption, previous medical history, pre-therapy laboratory test.

NRS-2002 score(s) and nutritional status of all patients were assessed at three-time points: pre-RT (T1, within five days of the start of radiotherapy), in the middle of radiotherapy (T2, $15 \pm 5$ radiotherapy times), and at the end of radiotherapy( $\mathrm{T} 3$, within 5 days of the end of radiotherapy).

\section{Follow-up}

Follow-up duration was measured from the day of diagnosis to the last visit or death. Conventional follow-up included physical examinations, head and neck MRI or CT, plasma Epstein-Barr virus DNA, 
thyroid function, abdominal sonography, chest radiography or CT, whole bone scan, and PET-CT if necessary, at least 3-6 months during the first two years, and then every 6-12 months.

\section{Statistical analysis}

Continuous variables were expressed as mean value and range; categorical variables were expressed as counts and percentages. The McNemar Test was used to test for statistically significant in the frequency of malnutrition between different time points. A comparison of complication rates within well-nourished and malnourished patients was performed using the Pearson Chi-square test. The Progress-free survival (PFS, from first therapy to recurrence or metastases or death), overall survival (OS, from the date of beginning therapy to the date of death for any cause) was performed using the Kaplan-Meier method to compare survival outcomes, and differences were evaluated using the log-rank test. Cox proportional hazards model was used to estimate hazard ratios (HR), 95\% confidence intervals (CI) in multivariate analysis. Two-sided $p$ values of $<0.05$ were considered statistically significant. All statistical analyses were performed using SPSS version 24.0 software for windows

\section{Results}

\section{Summarization of baseline information of patients}

An ensemble of 113 NPC patients was analyzed, and corresponding data were collected related to the elementary parameters used to determine the association between malnutrition and physical appearances. According to GLIM criteria, 19 of 113 NPC patients (16.8\%) before the radiotherapy went into malnutritional cohorts, and $83.2 \%$ of patients were well-nourishment. The baseline characters were shown in Table 1. The prevalence of malnutrition according to GLIM criteria significantly correlates with overall stage and induction chemotherapy ( $p=0.017$, and $p=0.002$, respectively).

\section{The dynamic of nutritional status evaluated by GLIM criteria along with radiotherapy}

NPC patients with malnutrition assessed by GLIM criteria during the whole process of radiotherapy was shown in Table 2. 16.8\% of patients were malnutrition before radiotherapy, however, $91.2 \%$ were malnutrition at the end of radiotherapy, which exhibiting an increased prevalence of malnutrition with the radiotherapy process. Besides nutritional status, according to GLIM criteria, we also investigated the phenotypic and etiologic criteria of GLIM criteria. Aiming to comparing the efficacy of different phenotypic criteria, we sought to use the different combinations by two parameters belonging to phenotypic and etiologic criteria. Both strategies were exercised to assess the nutritional status of NPC patients at different times during the whole process of radiotherapy. Therefore, ten subgroups were constructed with combination of every two indexes belonging to phenotypic and etiologic legs respectively, which was shown in Table 3. The result remained consistent with that evaluated by GLIM criteria, and differed phenotypic and etiologic criteria made no difference in the result.

Even though all the phenotypic criteria combining with different etiologic criteria lead to the same malnutritional trend along with radiotherapy, the incidence of malnutrition assessed by differed 
phenotypic criteria varied according to the results above, indicating that different phenotypic criteria perform the distinguished sensitivity and specificity. From Table 3, at two-time points (T2 and T3), weight loss $>5 \%$ combined with reduced food intake or disease burden led to the highest proportion of malnutrition, and the lowest proportion was from low BMI combined with reduced food intake or disease burden. Notably, for the two muscle loss criteria, FFMI was more sensitive than ASMI, regardless of different combined etiologic criteria and different time points ( $T 1, T 2$ and $T 3)$, which was concluded from the higher prevalence of malnutrition by low FFMI compared to ASMI.

\section{The pre-radiotherapy FFMI and ASMI in phenotypic GLIM criteria associated with radiotherapy-related adverse effects}

Since the malnutrition associates with the multiple conditions or factors of the NPC patients and progressively descends to the worsened situations, we next investigated the potential association between the pre-radiotherapy phenotypic GLIM criteria and adverse effects. Noteworthily, there was no significant correlation found between malnutrition evaluated by GLIM criteria. However, despite of unfound association between GLIM criteria and malnutrition, an altered view toward the single phenotypic GLIM criterion opened a new discovery to our study. It has been emphasized that GLIM criteria encapsulate the multiple sections, disparate criteria skid to distinct characteristics, each of which entails the certain physical status of the patient, indicating it might lead the statistical analysis to different results. Our result was consistent with this prediction entirely; different GLIM criteria performed differently when we endeavored to link every phenotypic criterion to post-treatment adverse effects.

In our study, no grade 4 non-hematologic toxicity was observed, and 22 cases (19.5\%) were received parenteral nutrition during radiotherapy. The most common acute toxicities were nausea, mucositis, and dermatitis. Grade $\geq 2$ nausea, mucositis, dermatitis occurred in 94 patients (83.2\%), 81 patients $(71.7 \%)$, 72 patients $(63.7 \%)$, respectively (Table $\mathbf{S 1})$.

With regards to the correlational analysis, the NPC patients with low FFMI statistically possessed a high incidence of mucositis $(P=0.004)$, radiodermatitis $(p=0.019)$ (Table 4). Additionally, low ASMI rendered corresponding patients more susceptible to mucositis (94.7\% vs. $67.0 \%, p=0.014)$ (data not are shown). Apart from the multiple adverse events, parenteral nutrition supplement was necessitated for the NPC patients with low BMI $(60.0 \%$ vs. $15.5 \%, p=0.003)$ and FFMI $(35.7 \%$ vs $14.1 \%, p=0.012)$ (data not are shown). The relationship between low FFMI and adverse effects were shown in Table 4.

Given that ensuing adverse effects following radiotherapy was a definite threat for extra-hospital life quality of NPC patients, prophylactic efforts would become more available and purposive predicated on the exposition upon the built-up association between pre-radiotherapy nutritionally diagnostic parameters and post-treatment performance by our study.

\section{Malnutrition was related to poor survival}


With an end to the analysis adverse events, consequentially, NPC patients' survival period linking to malnutrition determined by GLIM criteria was inspected. With a median follow-up of 28.3 months (range 6.2-43.8 months), ten patients died: four from tumor progress, three from hemorrhage in nasopharyngeal, two from complications, and one because of myocardial infarction. Nineteen patients experienced treatment failures: four were locoregional, 14 were metastatic, and one suffered from both.

For all patients, the 2-year PFS and OS were $81.1 \%$ and $91.8 \%$, respectively. Univariate analysis, taking age ( $<60$ years old vs. $\geq 60$ years old), gender, ECOG, overall stage, induction chemotherapy, NRS-2002 before radiotherapy and nutritional status before radiotherapy as prognostic variables for 2-year PFS and OS, was performed using the log-rank test. Gender, overall stage, and nutritional status before radiotherapy were significant predictors of 2-year PFS ( $p=0.028, p=0.018, p=0.015$, respectively). Multivariate analysis showed that gender and nutritional status were independent predictors of PFS, which confirmed that malnutrition would lead to poor survival in NPC patients when the confounding factors were considered and modified by multivariate analysis $₫$ Table $5 \rrbracket$.

The correlation between different groups of nutritional status and survival was shown in Figure 1a. 2-year PFS was $88.9 \%$ in the well nutrition group vs $62.1 \%$ in the malnutrition group $(\mathrm{HR}, 2.964 ; 95 \% \mathrm{Cl}, 1.299$ 8.834; $p=0.013)$. However, OS failed to act similarly for the construction of association with malnutrition (94.7\% vs. $91.0 \%, p=0.882)$, which was shown in Figure 1b.

\section{Discussion}

Based on the previous study, radiotherapy acts to devastate the nutritional status of patients, portending unfavorable post-treatment outcomes for cancer patients [20]. Especially for NPC patients the nutritional defect possesses a high prevalence and negatively disrupts the clinical performance of NPC patient. Therefore, it necessitates the general assessment of the nutritional status of NPC patients. GLIM criteria, as a well-popularized strategy to assess the nutritional status, get reasonably engaged with our study targeting NPC, extracting valued clinical guidelines for nutritional management and practice. Likewise, insightful research into this project may provide more evidence allowing for usability and effectiveness to GLIM criteria.

Undoubtedly, cancer, including NPC, characterize themselves as a cohort of consumptive diseases, which competitively and rapaciously seizure the nutrition due to the enormous energy demands resulting from uncontrollable self-reproduction and proliferation, as a result, heavily uneven distribution of nutrition tilted to tumor cells sparks the consumption of nutrition for cancer patients [21], rendering it understandable that the advanced stage of NPC accompanies the malnutrition before treatment from our result.

According to several reports resembling our study, advanced NPC stage cancer patients are more likely to experience weight loss $[10,22]$. Conceivably, locoregionally advanced tumor progresses for a long period, concurrent with wide deterioration and corresponding symptoms (like loss of appetite, swallowing, and chewing problems) featuring the impediment to caloric intake, which in the end plunges into nutrition shortage and induced malnutritional ultimately. Our study has uncovered the risk factors, including $\mathrm{N}$ 
stage, overall stage, and IC leading to malnourishment in the clinical practice, allowing for more sophisticated and reasonable managements or nutritional intervention in a preventive fashion.

As for the impact of IC on nutritional status, as an efficacious method to combat the tumor metastasis, it plays an indispensable role especially for advanced stage NPC when noting that $53.9 \%$ of NPC patients with IVA, and about $52.2 \%$ of patients received IC treatment as a necessary strategy in our study. In one sense, IC prolonged the survival of NPC patients $[23,24]$. Nonetheless, the concomitant increased ICinduced toxicity much more likely courts the patients' malnutrition simultaneously, which acts as a double-edged sword. Therefore, to balance the side effects and efficacy has been up to the agenda, improved IC treatment bearing more effectiveness and less toxicity is supposed to be considered and developed. Instructionally speaking, prior assessment of nutritional status and examination of nutritional signs for the NPC patients are ineluctably required. Early nutritional support such as enteral or parenteral nutrition figures as a strongly-recommended strategy before radiotherapy lest more difficult situations occur to the patients.

According to the previous studies [4, 12], radiotherapy undermines nutrition status, our result of increased prevalence of malnutrition on NPC along with radiotherapy conforms to this observation. The disruptive effect on the tumor and normal cells from radiotherapy incentivizes the impedimental process, leading to the patients' physical feebleness. On the other hand, released substrates or chemicals from shattered cells and exposed antigens on the targeted cells induce immune responses and inflammation, leading to conclusively the high prevalence of malnutrition during radiotherapy [25-27]. Therefore, radiotherapy would aggravate the NPC patients' nutritional status unmistakably, and the careful management of NPC patients' nutrition appeared critical and indispensable in the case declined nutritional status concomitant with radiotherapy heavily compromise the outcomes of patients.

According to the GLIM criteria, if there is one criterion belonging to phenotypic and etiologic criteria respectively, it will denote malnutrition. Compared to a simple parameter or tool used to assess the nutritional status, GLIM criteria to some degree cover the specific information depicting the differed characteristics of patients; therefore, it might be more comprehensive. The different combinations of phenotypic GLIM criteria, weight loss, BMI, ASMI, and FFMI, demonstrated the polarized incidence of malnutrition. Low BMI contributed to the least proportion of malnutrition meaning the lowest sensitivity. On the other hand, weight loss $>5 \%$ contributed to maximum proportion displaying the highest sensitivity and lowest specificity, and FFMI generally held the second place in sensitivity.

However, weight alteration as a standard to assess the nutritional status displays the shortcomings and becomes easily influenced by many confounding factors because of its widely embraced contents, for an instance, loss of skeletal muscle and increased adipose tissue would possibly amount to the stable weight, masking the objective situations [28]. Therefore, instead of the simple weight-related evaluation, body composition parameters, such as FFM and ASM, release more precise and relevant information to detect and monitor nutritional status [29]. In contrast to weight evaluation, FFMI stays relatively impervious to many other factors and holds considerable sensitivity. Taking clinical practice for 
nutritional management into account, patients who keep under the malnutritional risk must be screened out and monitored closely as early as possible. Missing any patient with malnutritional risk would be disfavored, therefore, FFMI, compared to other criteria, is of much practicality, to spot as many patients under malnutrition as possible.

Utilization of an effective method to improve FFM and FFMI before radiotherapy may reduce the therapy complications. Nutrition and exercise are the two main ways to improve FFM and FFMI [30]. Sufficient energy supply was able to maintain skeletal muscle mass during radiotherapy. Therefore, rational and effective dietary advice and nutrition intervention should be considered. Besides, physical exercise is another approach to gain skeletal muscle. Lonbro et al.[31]demonstrated that resistance of training effectively increased lean body mass in head and neck cancer patients undergoing radiotherapy. However, there is no long-term intervention and optimal diet guidance to maintain SM and compensate the metabolic consumption for NPC patients with low FFMI before radiotherapy.

Nutritional status involves inseparably with the cancer prognosis and subsequent progress, as evidenced in several studies that weight loss portends the poor outcomes of patients to some extent [32, 33]. Essentially, malnutrition evidently causes the decline of patient life-related conditions including the metabolic levels represented by anabolic hormones and tolerance to diseases due to compromised immunity, which associates with the constant downregulation of released inflammatory cytokines. As a result, patients with malnutrition become incompetent to cancer progression and suffer from the shortened survival period [34, 35]. Hong et al. [11] assessed the nutritional status by modified nutritional index, consequently elucidating that malnutrition relates to worse 5 -year OS $(61.8 \%$ vs $77.1 \%, p=0.02)$. Also, a meta-analysis study from another angle revealed the potential concatenation binding malnutrition and survival, indicating that NPC patients with low prognostic nutritional index (PNI) had worse PFS and OS [36]. Partly Consistent in our study, malnutrition of NPC patients contributed to shorter PFS, and meaningfully, advanced efforts are required for the more in-depth research on survival period and malnutritional status. Notwithstanding, the consistent finding of the relationship between malnutrition and OS failed to show in our study, which is presumably ascribed to the inadequate follow-up duration which should have been longer because NPC patients have a long survival period.

We have to admit that several limitations in our study. Evidently, our study belongs to the retrospective study and some information is likely to be omitted or disinformed, instead all relevant data would be closely observed and collected in prospective study. For an instance, C-reaction protein, which denotes the inflammation state counted in etiologic criteria of GLIM criteria, unfortunately appeared missing in our study due to its unnecessariness in radiotherapy department. Besides, insufficient number of patients collected may also affect the results, which would otherwise drive the outcomes more significant like in survival analysis and unveil some other hidden findings.

\section{Conclusion}


The malnutrition according to GLIM criteria is comparatively prevailing and the prevalence gets increasing by the times of radiotherapy. Among the phenotypic criteria in GLIM, several adverse effects are linked to ASMI and FFMI. More importantly, based on our study, the declined nutritional status of NPC patients would negatively affect the PFS. In a nutshell, our findings confirm that GLIM could be used to assess nutritional status in NPC patients and have potential clinical use in predicting which cohorts of patients are susceptible to poor nutritional status, and provide new clues for appropriate nutritional diagnosis and comprehensive nutritional management of patients with NPC.

\section{Declarations}

\section{Ethics approval and consent to participate}

The project was approved and supervised by the Ethics Committee of the Beijing Cancer Hospital \& Institute, Peking University School of Oncology (Document Number: 2020YJZ72).

\section{Consent for publication}

Not applicable.

\section{Availability of data and materials}

The datasets generated and/or analyzed during the current study are not publicly available because they contain personal information but are available from the corresponding author upon the reasonable request.

\section{Competing interests}

The authors declare that they do not have any conflicts of interest

\section{Funding}

This research received the funding from WU JIEPING Medical Foundation for clinical research. Foundation number冈320.6750.17177.

\section{Author contributions}

All the authorship contributed to the work and approved the final version of the manuscript. Conception and study design, development of methodology: LQ and SY;

Data collection, formal analysis, writing - original draft, review \& editing: WM and ZLC;

Data collection, writing - review \& editing, supervision: CCS;

Data analysis and assistance to manuscript writing: ZD; 
Data check, modification: ZBM, XSW, LWX, XXL and WWH;

Manuscript revision: WYJ, ZB, ZT and JSL.

\section{Acknowledgements}

No applicable in this section.

\section{Author details}

${ }^{1}$ Key Laboratory of Carcinogenesis and Translational Research (Ministry of Education/Beijing), Department of Radiation Oncology, Peking University Cancer Hospital and Institute, Beijing 100142, People's Republic of China

2 Division of Medical \& Surgical Nursing, School of Nursing, Peking University, 100191, Beijing, China

${ }^{3}$ Department of Radiation Oncology, the First Hospital of Tsinghua University, No.6 Jiuxianqiao 1st Road, Chaoyang District, Beijing, 100016, China.

\section{References}

1. Bray F, Ferlay J, Soerjomataram I, Siegel RL, Torre LA, Jemal A. Global cancer statistics 2018 : GLOBOCAN estimates of incidence and mortality worldwide for 36 cancers in 185 countries. CA: a cancer journal for clinicians. 2018;68(6):394-424.

2. Sun X, Su S, Chen C, Han F, Zhao C, Xiao W, et al. Long-term outcomes of intensity-modulated radiotherapy for 868 patients with nasopharyngeal carcinoma: an analysis of survival and treatment toxicities. Radiotherapy and oncology : journal of the European Society for Therapeutic Radiology and Oncology. 2014;110(3):398-403.

3. Lee N, Harris J, Garden AS, Straube W, Glisson B, Xia P, et al. Intensity-modulated radiation therapy with or without chemotherapy for nasopharyngeal carcinoma: radiation therapy oncology group phase II trial 0225. Journal of clinical oncology : official journal of the American Society of Clinical Oncology. 2009;27(22):3684-90.

4. Hong JS, Wu LH, Su L, Zhang HR, Lv WL, Zhang WJ, et al. Effect of chemoradiotherapy on nutrition status of patients with nasopharyngeal cancer. Nutrition and cancer. 2016;68(1):63-9.

5. Oates JE, Clark JR, Read J, Reeves N, Gao K, Jackson M, et al. Prospective evaluation of quality of life and nutrition before and after treatment for nasopharyngeal carcinoma. Archives of otolaryngology--head \& neck surgery. 2007;133(6):533-40.

6. Li G, Jiang XY, Qiu B, Shen LJ, Chen C, Xia YF. Vicious circle of acute radiation toxicities and weight loss predicts poor prognosis for nasopharyngeal carcinoma patients receiving intensity modulated radiotherapy. Journal of Cancer. 2017;8(5):832-8. 
7. Mallick S, Benson R, Rath GK. Radiation induced oral mucositis: a review of current literature on prevention and management. European archives of oto-rhino-laryngology : official journal of the European Federation of Oto-Rhino-Laryngological Societies (EUFOS) : affiliated with the German Society for Oto-Rhino-Laryngology - Head and Neck Surgery. 2016;273(9):2285-93.

8. Capuano G, Grosso A, Gentile PC, Battista M, Bianciardi F, Di Palma A, et al. Influence of weight loss on outcomes in patients with head and neck cancer undergoing concomitant chemoradiotherapy. Head \& neck. 2008;30(4):503-8.

9. Takenaka Y, Takemoto N, Nakahara S, Yamamoto Y, Yasui T, Hanamoto A, et al. Prognostic significance of body mass index before treatment for head and neck cancer. Head \& neck. 2015;37(10):1518-23.

10. Peng $H$, Chen BB, Tang LL, Chen L, Li WF, Zhang $Y$, et al. Prognostic value of nutritional risk screening 2002 scale in nasopharyngeal carcinoma: A large-scale cohort study. Cancer science. 2018;109(6):1909-19.

11. Hong JS, Hua YJ, Su L, Zhang HR, Lv WL, Chen XY, et al. Modified-Nutrition Index is a Significant Prognostic Factor for the Overall Survival of the Nasopharyngeal Carcinoma Patients who Undergo Intensity-modulated Radiotherapy. Nutrition and cancer. 2017;69(7):1011-8.

12. Irungu CW, Oburra HO, Ochola B. Prevalence and Predictors of Malnutrition in Nasopharyngeal Carcinoma. Clinical medicine insights Ear, nose and throat. 2015;8:19-22.

13. Ding H, Dou S, Ling Y, Zhu G, Wang Q, Wu Y, et al. Longitudinal Body Composition Changes and the Importance of Fat-Free Mass Index in Locally Advanced Nasopharyngeal Carcinoma Patients Undergoing Concurrent Chemoradiotherapy. Integrative cancer therapies. 2018;17(4):1125-31.

14. Rasmussen $\mathrm{HH}$, Holst $\mathrm{M}$, Kondrup J. Measuring nutritional risk in hospitals. Clinical epidemiology. 2010;2:209-16.

15. Arends J, Bachmann P, Baracos V, Barthelemy N, Bertz H, Bozzetti F, et al. ESPEN guidelines on nutrition in cancer patients. Clinical nutrition (Edinburgh, Scotland). 2017;36(1):11-48.

16. Galindo Martín CA, Aportela Vázquez VA, Becerril Hernández F, Aguilar Medina CR, Ayala Carrillo SL, Chávez Flores $A$, et al. The GLIM criteria for adult malnutrition and its relation with adverse outcomes, a prospective observational study. Clinical nutrition ESPEN. 2020;38:67-73.

17. Cederholm T, Jensen GL, Correia M, Gonzalez MC, Fukushima R, Higashiguchi T, et al. GLIM criteria for the diagnosis of malnutrition - A consensus report from the global clinical nutrition community. Clinical nutrition (Edinburgh, Scotland). 2019;38(1):1-9.

18. Kondrup J, Allison SP, Elia M, Vellas B, Plauth M. ESPEN guidelines for nutrition screening 2002. Clinical nutrition (Edinburgh, Scotland). 2003;22(4):415-21.

19. Kondrup J, Rasmussen HH, Hamberg O, Stanga Z. Nutritional risk screening (NRS 2002): a new method based on an analysis of controlled clinical trials. Clinical nutrition (Edinburgh, Scotland). 2003;22(3):321-36.

20. Arends J, Bachmann P, Baracos V, Barthelemy N, Bertz H, Bozzetti F, et al. ESPEN guidelines on nutrition in cancer patients. Clinical nutrition (Edinburgh, Scotland). 2017;36(1):11-48. 
21. Arends J, Baracos V, Bertz H, Bozzetti F, Calder PC, Deutz NEP, et al. ESPEN expert group recommendations for action against cancer-related malnutrition. Clinical nutrition (Edinburgh, Scotland). 2017;36(5):1187-96.

22. Chen YJ, Chen SC, Wang CP, Fang YY, Lee YH, Lou PJ, et al. Trismus, xerostomia and nutrition status in nasopharyngeal carcinoma survivors treated with radiation. European journal of cancer care. 2016;25(3):440-8.

23. Sun Y, Li WF, Chen NY, Zhang N, Hu GQ, Xie FY, et al. Induction chemotherapy plus concurrent chemoradiotherapy versus concurrent chemoradiotherapy alone in locoregionally advanced nasopharyngeal carcinoma: a phase 3 , multicentre, randomised controlled trial. The Lancet Oncology. 2016;17(11):1509-20.

24. Zhang Y, Chen L, Hu GQ, Zhang N, Zhu XD, Yang KY, et al. Gemcitabine and Cisplatin Induction Chemotherapy in Nasopharyngeal Carcinoma. The New England journal of medicine. 2019;381(12):1124-35.

25. Stone HB, Coleman CN, Anscher MS, McBride WH. Effects of radiation on normal tissue: consequences and mechanisms. The Lancet Oncology. 2003;4(9):529-36.

26. Mahdavi R, Faramarzi E, Mohammad-Zadeh M, Ghaeammaghami J, Jabbari MV. Consequences of radiotherapy on nutritional status, dietary intake, serum zinc and copper levels in patients with gastrointestinal tract and head and neck cancer. Saudi medical journal. 2007;28(3):435-40.

27. Jager-Wittenaar H, Dijkstra PU, Vissink A, Langendijk JA, van der Laan BF, Pruim J, et al. Changes in nutritional status and dietary intake during and after head and neck cancer treatment. Head \& neck. 2011;33(6):863-70.

28. Prado CM, Siervo M, Mire E, Heymsfield SB, Stephan BC, Broyles S, et al. A population-based approach to define body-composition phenotypes. The American journal of clinical nutrition. 2014;99(6):1369-77.

29. Cederholm T, Bosaeus I, Barazzoni R, Bauer J, Van Gossum A, Klek S, et al. Diagnostic criteria for malnutrition - An ESPEN Consensus Statement. Clinical nutrition (Edinburgh, Scotland). 2015;34(3):335-40.

30. Trouwborst I, Verreijen A, Memelink R, Massanet P, Boirie Y, Weijs P, et al. Exercise and Nutrition Strategies to Counteract Sarcopenic Obesity. Nutrients. 2018;10(5).

31. Lonbro S, Dalgas U, Primdahl H, Johansen J, Nielsen JL, Aagaard P, et al. Progressive resistance training rebuilds lean body mass in head and neck cancer patients after radiotherapy-results from the randomized DAHANCA 25B trial. Radiotherapy and oncology : journal of the European Society for Therapeutic Radiology and Oncology. 2013;108(2):314-9.

32. Zeng Q, Shen LJ, Guo X, Guo XM, Qian CN, Wu PH. Critical weight loss predicts poor prognosis in nasopharyngeal carcinoma. BMC cancer. 2016;16:169.

33. Shen LJ, Chen C, Li BF, Gao J, Xia YF. High weight loss during radiation treatment changes the prognosis in under-/normal weight nasopharyngeal carcinoma patients for the worse: a retrospective analysis of 2433 cases. PloS one. 2013;8(7):e68660. 
34. Gundog M, Basaran H. Pretreatment low prognostic nutritional index and low albumin-globulin ratio are predictive for overall survival in nasopharyngeal cancer. European archives of oto-rhinolaryngology : official journal of the European Federation of Oto-Rhino-Laryngological Societies (EUFOS) : affiliated with the German Society for Oto-Rhino-Laryngology - Head and Neck Surgery. 2019;276(11):3221-30.

35. Su L, Lin Q, Li R, Hua Y, Zhang H, Song X, et al. Prognostic value of nutritional impairment on treatment-related toxicity and survival in patients with nasopharyngeal carcinoma taking normal nutrition before radiotherapy. Head \& neck. 2020.

36. Tu X, Ren J, Zhao Y. Prognostic value of prognostic nutritional index in nasopharyngeal carcinoma: A meta-analysis containing 4511 patients. Oral oncology. 2020;110:104991.

\section{Tables}

Due to technical limitations, table $1,2,3,4$ and 5 is only available as a download in the Supplemental Files section.

\section{Figures}


a

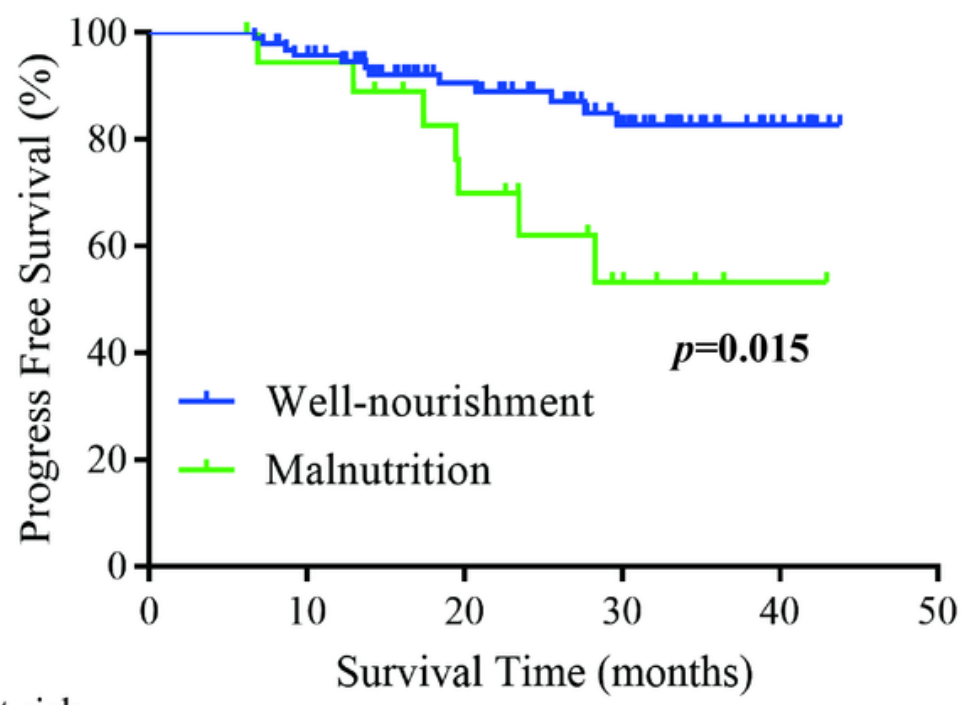

Patients at risk

$\begin{array}{ccccccc}\text { Well-nourishment } & 94 & 89 & 58 & 36 & 10 & 1 \\ \text { Malnutrition } & 19 & 18 & 12 & 6 & 2 & 1\end{array}$

b

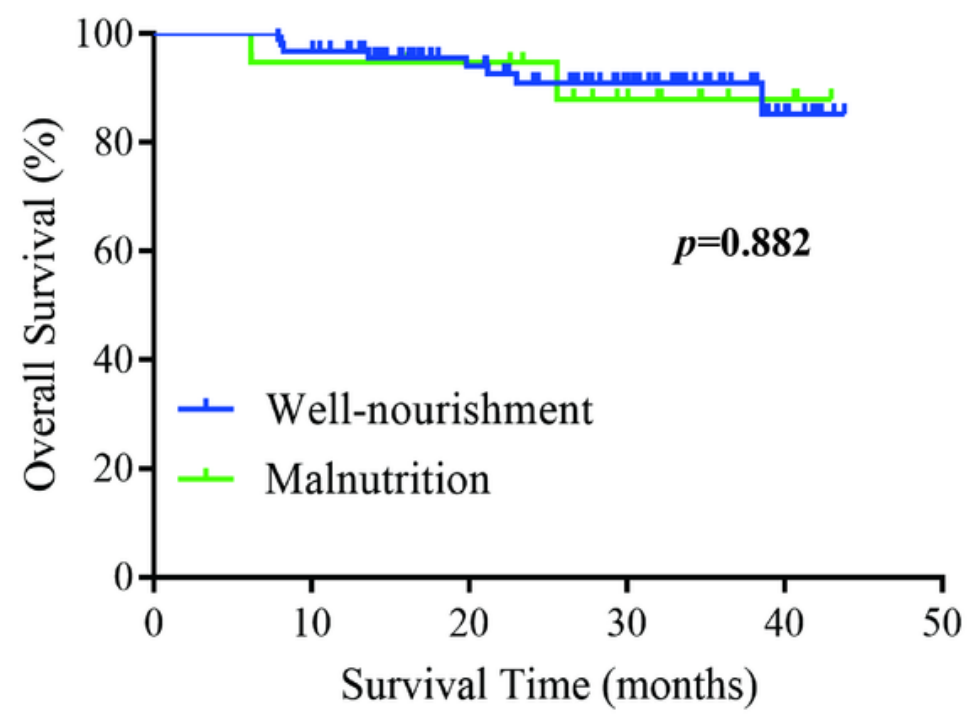

Patients at risk

$\begin{array}{ccccccc}\text { Well-nourishment } & 94 & 92 & 64 & 42 & 12 & 1 \\ \text { Malnutrition } & 19 & 19 & 17 & 11 & 4 & 1\end{array}$

\section{Figure 1}

Survival analysis including PFS and OS by Kaplan-Meier method with nutritional status of NPC patients a. Kaplan-Meier survival curve between malnutrition and PFS $(p=0.015)$ b. Kaplan-Meier survival curve between malnutrition and OS $(p=0.882)$

\section{Supplementary Files}


This is a list of supplementary files associated with this preprint. Click to download.

- Table1.tif

- Table2.tif

- Table3.tif

- Table4.tif

- Table5.tif

- TableS1.tif 